\title{
Plateforme de Michel Houellebecq. «Au milieu du monde», je vois et je hais.
}

\author{
Katerine Gagnon
}

\begin{abstract}
Michel Houellebecq's latest novel, Platform, brings up a certain urgency of criticism not so much by its anti-democratic ideas portrayed by the author, but by the problematic strategy of non-adhesion by which the narrator guarantees his impunity. Indeed, the narrator claims to be a passive observer «in the midst of the world». In truth, Michel is a parasitical figure who, animated by a purely cynical will, feeds on organisms whose demise he anticipates and even brings to an end. The novel becomes a space for hatreds and their own apocalyptic exhaustion: the hate and the end of the Western world, of Islam, of women and, finally, of the individual. Instead of handing literature over to a lucid knight fighting for the truth, Platform serves the desire of a man who, having nothing to offer to others, wishes to disappear and sabotages the hopes of all humanity.
\end{abstract}

\author{
La possibilité de vivre \\ Commence dans le regard de l'autre \\ Tes yeux $m$ 'aspirent et je m'enivre \\ Je me sens lavé de mes fautes \\ Michel Houellebecq, Renaissance.
}

Si Houellebecq fait œuvre de vision - vision du monde, sur lui et à travers lui son adhésion à ce qu'il dit voir et à ce qu'il en dit n'a rien de transparent. C'est moins sa parole elle-même qui pose problème que la stratégie à laquelle elle répond: Michel, le narrateur de Plateforme, s'il porte le nom de l'auteur, ne reconnaît guère la création dont il est lui-même l'auteur, et du reste il parle avec si peu de conviction que le lecteur a peine à retrouver la place que Houellebecq entend occuper dans sa parole comme dans le monde.

La vérité est que Michel ne croit en rien et que le lecteur ne doit pas le croire. Son cynisme n'a rien de résigné, il n'a rien à voir avec un certain courage de la lucidité. Au contraire, il est opportuniste et décadent. Celui qui prétend ne pas vouloir comprendre comment la misère est venue au monde occidental explique pourtant moins son état, son fonctionnement actuels qu'il en précipite la fin.

On comprendra que l'enjeu est de taille: il s'agit, ni plus ni moins, du destin de ces valeurs dont est constituée l'identité de notre Occident démocratique. Notre époque, en crise, tente intensément de penser la perversion des 
idéaux d'égalité, de liberté, de même que des promesses de la démocratie; or, prenant au mot ces défaillances, le cynisme de Michel ne fait pas que penser les perversions, il les agit et les mène à leur propre épuisement. C'est le cynisme du parasite qui se nourrit d'un organisme dont il anticipe et achève l'écroulement, la fin, dans un désir indécent de disparition et d'indifférenciation des instances d'autorité.

Je crois donc à une certaine urgence de la critique. Il ne s'agit pas pour moi de relancer les polémiques déjà démarrées autour du livre, encore moins de (re)demander à l'auteur de rendre des comptes sur ses idées en dehors du livre, mais plutôt de prendre position en face du livre. Il s'agit de réfléchir les points aveugles et l'irréfléchi d'un livre qui prétend penser le monde, de résister à un réalisme de mauvaise foi qui, non content de représenter et de mettre en scène les différentes violences et aberrations contemporaines, en multiplie les lieux sans tout élucider. Racisme, misogynie, misanthropie dessinent une carte du réel de telle sorte que le roman se fait le terroir où germent toutes les haines. Non seulement le bonheur mais l'existence même de la race humaine doivent être défendus.

\section{La haine de l'Occident}

Michel est le produit dégénéré d'un espace occidental qui reste, avant tout, une terre de luttes et d'injustices. Le capitalisme est «dans son principe un état de guerre permanente, une lutte perpétuelle qui ne peut jamais avoir de fin» (293) et la libéralisation de la sexualité a créé un système de lutte parallèle, au sein duquel l'individu est appelé à conquérir son droit de jouir d'un autre corps. Mais dans ces conditions, Michel perd à tous les coups. Il rêve donc d'une fin de la lutte, et en attendant l'avènement de l'indifférenciation qui en signera la fin, il tente de survivre en y échappant, comme un parasite.

L'ironie du statut économique de cet être soucieux, semble-t-il, de ne pas trop se dépenser est mordante. D'abord fonctionnaire paresseux, Michel fait figure du parasite salarié chargé de veiller à la survie d'un certain art contemporain, caractérisé dans le roman par un désengagement social et un narcissisme qui frisent l'indécence - et la caricature. Mais le roman commence ainsi: le père de Michel meurt. Celui-ci ne tarde pas, en fait, à connaître la paisible existence du rentier: le fils vivra dorénavant à même l'héritage du père, ce père conquérant et énergique qui avait réussi à tout gagner: "Il avait profité de la vie, le vieux salaud; il s'était démerdé comme un chef. "T'as eu des gosses, mon con... me dis-je avec entrain; t'as fourré ta grosse bite dans la chatte à ma mère."» (11)

Mais le père n'a rien légué de son «courage» ou de sa «virilité» à son fils fasciné, possédé, malgré tout, par «ce petit organe fendu, étrange»: «Mon enthousiasme pour les chattes n'avait pas décru, j'y voyais même un de mes derniers traits pleinement humains, reconnaissables; pour le reste, je ne savais plus très bien.» (68-69) Michel fait partie de ce monde où «les Occidentaux n'arrivent plus à coucher ensemble» (250), il participe de cette «misère sexuelle» dont il cherche moins à comprendre les causes que la logique de son fonctionnement. Car s'il explique l'incapacité des Occidentaux à s'aimer, à s'attacher à quelqu'un - ou leur «[inaptitude] à résumer l'ensemble de l'autre sexe à un seul être aimé» dirait le Michel d'Extension 
du domaine de la lutte (114) - par la faute des uns et des autres, c'est qu'en fait il n'a lui-même rien à offrir dans cette économie sexuelle en place (voir 146).

Rien n'attache le parasite à cet organisme occidental qui le nourrit (d'un point de vue économique du moins) sinon sa haine. L'Occident est ce qui le désigne comme être dégénéré, «indigne de vivre» - et parallèlement, l'existence même de Michel témoigne de la dégénérescence de cette civilisation, puisqu'elle n'a plus le courage d'éliminer son produit taré:

Européen aisé, je pouvais acquérir à moindre prix, dans d'autres pays, de la nourriture, des services et des femmes; Européen décadent, conscient de ma mort prochaine, et ayant pleinement accédé à l'égoïsme, je ne voyais aucune raison de m'en priver. J'étais cependant conscient qu'une telle situation n'était guère tenable, que des gens comme moi étaient incapables d'assurer la survie d'une société, voire tout simplement indignes de vivre. Des mutations surviendraient, survenaient déjà, mais je n'arrivais pas à me sentir réellement concerné...(308)

Michel annonce une double extinction; et du haut de sa plate-forme, il comprend quel parti il doit prendre: fuir la misère (sexuelle) de son terroir, exploiter celle (économique) des autres, et accélérer le destin apocalyptique du monde en favorisant une «professionnalisation de la sexualité» tout en la disant «inéluctable» (254). Le réalisme de Plateforme est donc traversé par la rhétorique cynique d'un narrateur sans volonté ni conviction, mais ce cynisme est en fait moins opportuniste que volontaire; en effet si Michel entend produire son état de paisible bonheur dans la misère ellemême, en se nourrissant d'elle, il souhaite aussi provoquer l'émergence des haines, et la fin de l'amour.

Pourtant l'enjeu est bien celui-là: bien plus qu'une vie sexuelle active et saine, il s'agit pour lui de trouver une femme à qui s'attacher. Qu'il s'agisse d'amour, je ne le crois point; il semble plutôt que Michel haïsse l'Occident précisément parce qu'en ce qui concerne la lutte sexuelle, il le condamne au parasitisme. Michel va donc vivre des restes du père par intermédiaire, dans le but ultime de s'accrocher à une «chatte» qui, seule, peut véritablement le sauver de l'annihilation dans le désespoir:

L'idée que j'étais solidaire de ce milieu ne m'avait jamais effleuré; c'était comme une atrophie chez moi, une absence. Il n'était pas certain que la société puisse survivre très longtemps avec des individus dans mon genre; mais je pouvais survivre avec une femme, $m$ 'y attacher, essayer de la rendre heureuse. (339, je souligne)

Ainsi le slogan du publi-reportage vantant les «avantages concurrentiels» (pour reprendre le titre de chapitre en question) de l'achat des prostituées thaïes pourrait bien être le sien: «I'm helping modern Western women to avoid what they despise.» (133) Encore qu'il lui faille des femmes «à la fois un peu mère de famille et un peu salope» (57), exactement comme sa mère. 


\section{Misogynie.}

Dans Plateforme, Michel participe à la mise en place d'un système d'exploitation, à la fois économique et sexuelle cette fois-ci, d'êtres humains davantage miséreux que les Occidentaux, soit les femmes du Tiers-Monde: «J'étais vaguement conscient, à ce moment, d'être à l'origine de quelque chose: sur le plan économique, j'étais certain d'avoir raison (...); mais je savais que les gens ont parfois du mal, étrangement, à accepter les idées simples.» (255) Or la simplicité dont il s'agit est celle d'une vérité amorale, économique, celle de la «simple» reconnaisse d'une «offre» ou d'un bassin de consommateurs qui existe déjà, et dont Michel luimême fait partic. Le projet à l'origine duquel il se trouve n'a donc d'autre fonction, dans sa logique cynique, que d'assurer une certaine disponibilité du sexe de la femme à l'homme. Car le sexe «sauve»l'homme du désespoir.

Source de plaisir permanente, disponible, les organes sexuels existent. Le dieu qui a fait notre malheur, qui nous a créés passagers, vains et cruels, a également prévu cette forme de compensation faible. S'il n'y avait pas, de temps à autre, un peu de sexe, en quoi consisterait la vie ? Un combat inutile contre les articulations qui s'ankylosent, les caries qui se forment. Tout cela, de surcroît, inintéressant au possible (...) (220-221)

La vie est, en soi, une lutte sans espoir. C'est le paradoxe du parasite qui, tout en luttant pour sa préservation, choisit de jouir, dans une dépense extrême, d'un corps inévitablement voué à la mort. Il faut pouvoir trouver des corps offerts à la jouissance, des corps généreux qui offrent leur plaisir en partage; il faut des êtres entièrement voués à la production de l'orgasme de l'autre, pour le moindre effort, sans la lutte, dans un climat de bonté apaisant. Contre l'impossibilité d'échapper à la mort, il faut compenser par la facilité d'une jouissance par laquelle l'homme déserté de Dieu peut encore connaître une sorte d'état de paix intérieure (169); et contre les bouches intelligentes qui produisent des discours sur le monde, qui expliquent et performent, à la fois, les combats du monde, il faut parfois préférer celle, gentille, qui se tait et reçoit, «une bouche bien chaude, prompte à avaler le sperme d'un ami véritable» (51).

Et c'est d'ailleurs bien des rapports de forces, à renverser, dont il est question dans Plateforme. Mais cette «amitié», cette admiration et cette solidarité ressenties pour la prostituée - «condamnée» comme lui, la Thaïe est la plus digne des femmes parce qu'elle «ne demandait honnêtement qu'à jouir de concert» comme lui (57) - , tous ces bons sentiments masquent un paternalisme, celui du colonisateur, animé des fantasmes les plus machistes et les plus dégradants à l'égard des femmes. Le discours tient finalement au suivant: l'Occidental salvateur apporte la liberté et l'autonomie aux déshéritées, la chance aux Sin du Tiers-Monde d'être à la fois de bonnes mères et des femmes jouissives, le choix aux nouvelles travailleuses du sexe mondial de devenir propriétaires d'ellesmêmes si elles désirent jouir de la vente de leur corps (335).

Il s'agit toutefois, en vérité, d'imposer un destin à la féminité, et d'y arriver ailleurs puisque c'est désormais impossible chez soi. Ce désir et ce 
savoir qui manquent aux Occidentaux - «offrir son corps comme un objet agréable, donner gratuitement du plaisir» - se réalisent pratiquement au mot grâce au tourisme sexuel qui systématise du reste «l'aliénation et la dépendance» qu'on tente d' «éviter» en Occident (254). Michel explique que l'état actuel de la misère sexuelle occidentale est «lié au narcissisme, au sentiment d'individualité», mais contrairement à ce qu'il dit, cela importe effectivement beaucoup ${ }^{\mathrm{i}}$. Car c'est précisément un destin de disparition, d'absorption dans le désir (déviant, pervers, surtout pédophile) des hommes que l'entreprise inaugurée par Michel scelle pour les femmes du Tiers-Monde, voire pour les Occidentales: si Michel est «à l'origine de quelque chose», c'est bien de morts, des morts symboliques et réelles.

Un combat hégélien de l'affirmation de soi sous-tend toute l'entreprise d'Aphrodite, si bien explicitée par son slogan: «Parce qu'on a le droit de se faire plaisir.» (266) On reconnaît dans le slogan publicitaire une réactualisation du mot d'ordre de mai ' 68 - une rappropriation cynique par les hommes puisqu'en fait il prend au mot la «loi de l'offre» strictement masculine et occidentale qui règle le nouvel ordre sexuel (et économique) (222-223) et agit bien plus qu'il ne prédit l'«expansion» de son «domaine» à toutes les femmes, y compris les Occidentales: «Ce qui va probablement se passer, c'est que les femmes deviendront de plus en plus semblables aux hommes. (...) Les femmes peuvent s'adapter aux valeurs masculines; elles ont parfois du mal, mais elles peuvent le faire, l'histoire l'a prouvé.» (154)

L'histoire que raconte Michel, quant à elle, prouve une chose: les heureux gagnants du tourisme sexuel (clients et professionnels) doivent mourir. Les destinations exotiques deviennent les domaines de la mort annoncée et perpétuée. Et en fait, les bains de sang dans lesquels s'éteint la chaîne Aphrodite rappellent désespérément le spectacle du gros vieillard moribond venu dilapider «ses dernières joies» auprès d'une jolie jeune Thaïe (113-114), ou encore le tableau dressé de Pattaya Beach, destination touristique préservée du cataclysme terroriste mais survivant comme «égout terminal où viennent aboutir les résidus variés de la névrose occidentale» (361), où l' "on ne vient pas pour refaire sa vie, mais pour la terminer dans des conditions acceptables» (362). Le bon TiersMonde, organisme débile et innocent, parasité en droit par les débris occidentaux, paradis terrestre des êtres voués autrement à la disparition et au désespoir, doit payer le prix pour tout le monde. Michel, en bon sauveur, ne leur épargne pas le mal.

\section{Haine de l'amour}

Michel recherche à la fois des «trous» où disparaître, comme avalé dans l'inconscience de l'orgasme (268), mais aussi des sexes par lesquels il accède au pouvoir de Dieu, devenant celui «dont dépend[ent] la sérénité et les orages» (169). Finalement, Michel rêve moins d'un honnête échange de plaisirs que de la certitude qu'il y aura toujours des «trous» où «gentiment vider ses testicules» (25), des corps desquels prendre un plaisir au moindre coût. 
Des femmes ouvertes, généreuses, il ne recherche et n'accepte, ne prend que cela: des failles faciles à habiter et des plaisirs faciles à recevoir, des «chattes» ouvertes, offertes (toutes ses amantes font preuve de cette sorte d'accueil: 90-91, $222,266,292)$. En échange de quoi Michel n'a rien à offrir: «il me semble que tu cherches quelque chose en moi, qui ne s'y trouve pas», dit-il à Valérie (146).

Or cette femme représente l'idéal de Michel, cet organisme consentant au parasite, sans volonté de domination ni de révolte; c'est la réalisation d'un fantasme d'indifférenciation au sein d'une autorité dégénérée, par défaut, la sienne. Valérie ne lui prend en effet rien, elle est entièrement don, générosité, bonté; elle est une «bonne prédatrice» (336) puisque «ce qui lui manquait, au fond, c'était surtout le désir de séduire» (65), une «prédatrice gentille» (337) puisque «jamais elle ne se retourn[e] contre» son amant (169). En vérité, bien qu'elle réussisse au plan professionnel, Valérie n'a rien d'une femme forte, dominante, libérée. C'est au contraire un sujet absorbé par Michel, «pareil» à lui dans ses désirs (230), ou plutôt entièrement voué à exaucer ceux de son amant (368): contre le démoniaque «vouloir» des femmes émancipées - celles qui ne veulent pas le bien de leur amant (entendre: la satisfaction complète et sans difficulté de leurs désirs; 133, 152) - Valérie ne veut rien de plus et rien de moi que Michel, tout en étant, heureusement, infiniment plus désirable que lui. De sorte que leur prétendu amour ne sert finalement qu'à entériner un usage, par Michel, de Valérie comme monnaie d'échange sur un marché sexuel devenu, grâce à son existence, littéralement échangiste.

En effet Michel partage le corps de son amante afin d'avoir le droit de jouir du corps de plusieurs femmes: sa nouvelle amante, une de ces rares «femmes qui éprouvent du plaisir, et qui ont envie d'en donner» (132), est généreuse à sa place et lui permet d'avoir accès à une économie sexuelle généreuse et en vérité hors de prix. Ainsi des femmes jugées parfaitement désirables selon les critères archétypaux côtoient des hommes moches avec lesquels Michel n'a de contact que par amantes interposées et échangées ${ }^{\mathrm{ii}}$. Par ailleurs Valérie, bisexuelle, exhibitionniste, libidinale, également clitoridienne et vaginale, permet à Michel d'étendre le territoire de sa jouissance au domaine de la pornographie: exhibitionnisme, échangisme, voyeurisme lesbien ${ }^{\text {iii }}$ et multiples combinaisons de partenaires s'enchaînent dans une logique de la surenchère non assumée.

En définitive, la virilité de Michel se déploie dans un climat de facilité paisible et invraisemblable qui n'a rien à voir avec la marchandisation de la sexualité annoncée et systématisée par la mise sur pied d'un réseau de tourisme sexuel. Si l' «amour sanctifie» $(90,123)$ la vie, c'est qu'il permet qu' «un instant de baise devienne éternité», pour reprendre l'heureuse expression de P. Varrod (Varrod, 109), et ce, sans que Michel n'ait à payer. Une vie sexuelle gratuite et sans problème d'aucune sorte. Un bonheur, aussi, qu'il ne mérite pas ${ }^{\mathrm{iv}}$ :

Je continuais à me demander ce que j'avais fait, au juste, pour mériter une femme comme Valérie. Probablement rien. Le déploiement du monde, me dis-je, je le constate; procédant empiriquement, en toute bonne foi, je le constate; je ne peux rien faire d'autre que le constater. (295) 
Michel ne fait rien, il laisse faire les autres. C'est tout ce qu'il sait (ou veut) faire. Il aime moins Valérie qu'il n'accepte sa dévotion; et puis il ne la tue pas, il laisse le monde s'entretuer et la prendre au passage. L'ironie de la fin, comme celle par laquelle commence le roman, tient à une mort dont il finit par se nourrir:

On peut certainement rester en vie en étant simplement animé par un sentiment de vengeance; beaucoup de gens ont vécu de cette manière. L'islam avait brisé ma vie, et l'islam était certainement quelque chose que je pouvais haïr; les jours suivants, je m'appliquait à éprouver de la haine pour les musulmans. J'y réussissais assez bien, et je recommençai à suivre les informations internationales. Chaque fois que $j$ 'apprenais qu'un terroriste palestinien, ou un enfant palestinien, ou une femme enceinte palestinienne, avait été abattu par balles dans la bande de Gaza, j'éprouvais un tressaillement d'enthousiasme à la pensée qu'il y avait un musulman de moins. Oui, on pouvait vivre de cette manière. (357)

Michel regarde le monde se venger à sa place. L'immonde, c'est ce dont il vit. On se demande finalement qui, du terroriste et du parasite, fait le plus de mal à l'environnement humain où il évolue.

\section{Discours de haine envers l'islam}

Le roman s'ouvre et se ferme sur deux meurtres commis par des Arabes, deux actes engendrés par un refus de l'Occident moins que par un choc avec celui-ci; la présence des Arabes et leurs gestes sont du reste en général interprétés par le discours informé de ceux qui ont subi de près la violence musulmane (Aïcha, l'Égyptien, le Palestinien). L'islam fait alors figure d'agent obscurantiste rétrograde et nuisible qui produit, dans son contact avec l'Occident, des sortes de monstres (30), de (religion déraisonnable» dont la volonté est de provoquer ou d'entretenir le mal sur terre (259-261), et en somme d'agent barbare qui tend à refuser le «paradis» sur terre à l'homme (358).

Et pourtant le meurtre du père et l'explosion à Krabi ne sont pas seulement des gestes par lesquels un fantasme - je l'appellerai moi aussi «celui de la pureté islamique» (Varrod, 104) - l'emporte sur celui de la mère-putain et de la marchandisation de la sexualité. Au contraire, il semble plutôt que le fantasme en jeu soit le même:

Le problème des musulmans, explique un Palestinien à Michel, c'est que le paradis promis par le prophète existait déjà ici-bas: il y avait des endroits sur cette terre où des jeunes filles disponibles et lascives dansaient pour le plaisir des hommes, où l'on pouvait s'enivrer de nectars en écoutant une musique aux accents célestes. (358)

La mise en marché des clubs Aphrodite misait également sur cette idée: ils offrent le paradis sur terre. L'acte terroriste est commis sous l'ordre d'une conviction religieuse qui reconnaît l'existence du mal incarnée ici sous cette forme. Cela dit 
il ne s'agit pas seulement d'un geste de survie qui témoigne des dernières convulsions d'un monde voué à une «évidente» extinction: «Pour lui il n'y avait aucun doute, explique le Palestinien, le système musulman était condamné: le capitalisme serait le plus fort.» (358 et 359) Avant que Michel puisse condamner l'islam, l'islam le condamne, car les Arabes, en résistant, en menant la lutte contre ce que j'ai appelé le fantasme de l'Occident, désignent aussi toute l'impuissance à quoi répond le projet cynique de Michel.

On l'a vu en effet, Michel est moins à l'origine de cette catastrophe finale (dans le roman du moins) qu'il en précipite l'avènement. C'est la seule chose qu'il puisse «créer»: la décadence, la fin des choses déjà inscrite dans leur mouvement. Pas étonnant donc qu'il prétende que sa haine se résorbe au moment où il reçoit du Palestinien l'idée réconfortante que le monde se chargera d'éliminer l'islam (358): non seulement le futur promet d'accomplir sa vengeance, mais Michel peut encore croire qu'il a précipité cette disparition-là en même temps que celles de l'Occident, des femmes et de Valérie. Pour lui tout est perdu, enfin dirait-on. Et en plus, il a trouvé des coupables à tous les coups. D'ailleurs le Michel de Plateforme ressemble à ce qui serait advenu du premier Michel, celui d'Extension du domaine de la lutte. Ce dernier utilise un pauvre minable, Tisserand, à la fois pour prouver mieux que lui-même la validité de sa théorie sur les deux libéralismes et leurs effets pervers, pour punir à sa place les femmes coupables du malheur des hommes et pour assouvir $\grave{a}$ sa place une tentation de meurtre que Michel est incapable d'assumer lui-même. Mais ce «désir» et cette «volonté de la lutte» reconnue chez Tisserand et manquante chez Michel mène le premier au suicide plutôt qu'au meurtre et laisse le second coincé avec la faillite de sa vie. À la fin de Plateforme, Michel se retrouve dans une semblable situation. Et s'il prétend que «l'absence d'envie de vivre, hélas, ne suffit pas pour avoir envie de mourir» (359), peut-être faut-il seulement comprendre qu'il attend encore, avant d'y penser, que «[son] livre touche à sa fin» (367).

\section{Haine de l'ego, haine de soi}

Les Occidentaux, les Thaïes et les Arabes également condamnés, Valérie disparue, que reste-t-il à faire? À toutes ces fins à la fois annoncées et souhaitées, Michel ne peut qu'y assister, et y survivre: comme témoin, il ne peut que témoigner de son impuissance, son incapacité à changer quoi que ce soit. Il ne peut donc plus qu'annoncer sa propre disparition; il ne lui reste que sa haine de l'ego, de lui-même. Parasite qui se nourrit des morts annoncées et annonce le mal dont il pourra, en parfait cynique, s'arranger, Michel a certes tout du monstre qui assume mal sa solidarité avec le monde d'où il est issu. Mais l'inverse est aussi vrai: tout juste après l'utopie des Particules élémentaires, ce rêve d'indifférenciation dans un amour platonique, anonyme et androgyne, c'est bien encore à la haine de l'ego que le roman se livre; encore une fois Houellebecq désigne, comme point aveugle et comme point de fuite de notre monde, «l'impasse de l'individualisme absolu et de l'affirmation de la différence pour elle-même» (Jourde, 276), la catastrophe à laquelle aboutit la surenchère de l'affirmation de soi. Et son personnage éponyme n'a ici nullement l'intention d'arrêter ce mouvement, il veut en vivre et en mourir. 
Michel écrit sa fin d'avance car il sait qu'à sa mort, rien ne lui survivra, qu'il n'y aura personne pour témoigner de lui: «Rien ne survivra de moi, je ne mérite pas que rien me survive; j'aurai été un individu médiocre, sous tous ses aspects. (...) Mon appartement sera loué à un nouveau résident. On m'oubliera. On m'oubliera vite.» (369-370) Le fils indigne du père n'en finira plus d'attendre sa mort, et il ne laissera, derrière et après lui, que les restes des restes du père, à l'usage d'autres heureux dépositaires - et le monde un petit peu (à peine) plus fou, plus horrible, plus laid qu'auparavant. Le cynique n'a rien à craindre de son sort; il pourra toujours trouver de quoi avoir à se contenter. Mais Michel laisse autre chose: «son» livre (367). Il lègue finalement une cuvre de mensonge et de haines; une littérature de taré, de moribond.

On se demande - avec le narrateur d'ailleurs - ce qu'il demeure de l'humanité dans ce roman traversé par les haines et par la parole d'un narrateur qui n'a rien à offrir à personne. Il est impossible, et peu pertinent d'ailleurs, de savoir quelle fut véritablement la volonté de l'auteur en écrivant ce roman dit autobiographique: mettre le lecteur en garde, le prévenir? Car certains arrivent à croire que Houellebecq «profondément c'est quelqu'un qui veut le bien de l'humanité» (Noguez, 21). Pourtant, je me demande quel espoir Houellebecq nous laisse. Peu importe qu'il prétende simplement voir et accepter (avoir le courage) de voir la laideur du monde, je sais que Michel - le narrateur - ment lorsqu'il voudrait frapper, que son mensonge accomplit sa haine. Et finalement, à vouer la littérature au mensonge et à la haine de cette manière, je me demande si Houellebecq lui-même croit encore à la littérature ou s'il désespère, en elle, de croire à un possible salut.

\section{Notes}

i «Il doit certainement se passer quelque chose, pour que les Occidentaux n'arrivent plus à coucher ensemble; c'est peut-être lié au narcissisme, au sentiment d'individualité, au culte de la performance, peu importe.» (250)

ii Voir, notamment, la scène où Jérôme et Michel discutent travail pendant que leurs femmes, échangées pour l'occasion, leur prodiguent une puissante fellation (267-268).

iii En effet, Michel joue le rôle du témoin ou du spectateur qui surprend le jaillissement d'un érotisme spécifiquement féminin (323), ou encore il surprend les baisers de Valérie avec les amantes qu'elle aura d'ailleurs été chercher elle-même, de sa propre initiative: les yeux fermés de Michel jouent le rôle d'une absence, d'une position à l'écart de la scène $(223,292)$.

iv Il faut bien comprendre l'incroyable rentabilité, pour Michel, de l'arrivée presque miraculeuse de Valérie, être exceptionnel et rare, dans sa vie: non seulement il n'a plus à dépenser (dépense économique) pour faire l'amour, mais il n'a plus à supporter non plus l'humiliation et l'échec (perte symbolique) que désigne la transaction économique (362). 


\section{Bibliographie}

Houellebecq, Michel. Extension du domaine de la lutte. Paris: J'ai lu, Éditions Maurice Nadeau, 1994.

- Les Particules élémentaires. Paris: Flammarion, 1998.

— Plateforme. Paris: Flammarion, 2001.

Jourde, Pierre. "L'individu louche: Michel Houellebecq.» La Littérature sans estomac. Paris: Pocket Agora, L'Esprit des Péninsules, 2002. 265-289.

Noguez, Dominic. Houellebecq en fait, cité par M.Prévost dans «Michel Houellebecq, contre le monde, pour la vie.» Spirale 198 (2004): 20-21.

Varrod, Pierre. «Michel Houellebecq: Plateforme pour l'échange des misères mondiales.» L'Esprit 11 (2001): 96-117. 